\title{
Elevated IL-6/IL-1Ra ratio as a prognostic biomarker of poor chemotherapy efficacy in Chinese patients with metastatic NSCLC, validated in a Caucasian patient cohort
}

\author{
FANG LIU ${ }^{1,2,6^{*}}$, BENOÎT SANSAS ${ }^{3 *}$, XAVIER PRÉVILLE ${ }^{3,7}$, ROMAIN GINESTE $^{1,2,8}$, \\ JIALEI WANG ${ }^{4,5}$, HUI YU ${ }^{4,5}$, XIA MENG ${ }^{1,2}$, ROMAIN MICOL ${ }^{3,9}$ and LUC BARRAUD ${ }^{3}$
}

${ }^{1}$ Transgene Biopharmaceutical Technology Co. Ltd.; ${ }^{2}$ Institut Mérieux Laboratory, Fudan University Shanghai Cancer Center, Shanghai 201315, P.R. China; ${ }^{3}$ Research and Innovation Department, Transgene S.A., 67405 Illkirch Graffenstaden, France; ${ }^{4}$ Department of Medical Oncology, Fudan University Shanghai Cancer Center, Shanghai 201315; ${ }^{5}$ Department of Oncology, Shanghai Medical College, Fudan University, Shanghai 200032, P.R. China

Received June 18, 2018; Accepted November 28, 2018

DOI: $10.3892 / \mathrm{mco} .2018 .1788$

\begin{abstract}
The treatment options for advanced (stage IV) non-small cell lung cancer (NSCLC) at diagnosis remain disappointing. The development of immunotherapeutic drugs may represent a promising alternative approach to the treatment of late-stage cancer at diagnosis. These current paradigms in cancer treatment highlight the need for new biomarkers related to the immune status of the patients and/or the tumor microenvironment, for immune as well as chemotherapeutic treatment options. The aim of the present study was to analyze soluble immune factors in patients with lung cancer treated with chemotherapy to identify prognostic biomarkers. For this purpose, the data obtained from two cohorts of patients from different clinical trials were analyzed: A Chinese patient cohort to identify potential prognostic biomarkers, and a validation cohort comprising patients with a similar clinical stage from a clinical trial in Europe. Analyses of soluble markers for inflammation and immune status were performed by standard assays and multiplex Luminex assays. Differences in overall survival (OS) and progression-free survival (PFS) were evaluated with the log-rank test and robustness was evaluated with the resampling approach. In the Chinese cohort,
\end{abstract}

Correspondence to: Dr Luc Barraud, Research and Innovation Department, Transgene S.A., 400 Bvd Gonthier d'Andernarch, 67405 Illkirch Graffenstaden, France

E-mail: barraud@transgene.fr

Present addresses: ${ }^{6}$ Cellular Biomedicine Group, Shanghai 201210, P.R. China; ${ }^{7}$ TALIX Therapeutics, 75000 Paris, France; ${ }^{8} \mathrm{Head}$ and Neck and Digestive Oncology, Astra Zeneca, 1702 Dilbeek, Belgium; ${ }^{9}$ Combined Therapeutics Inc., Boston, MA 02139, USA

*Contributed equally

Key words: immunophenotyping, prognostic factor, non-small cell lung cancer, cytokines four prognostic biomarkers of poor response to chemotherapy were identified, which had a significant impact on OS and PFS. It was confirmed in the Caucasian validation cohort that an increased value of the interleukin (IL)-6/IL-1Ra cytokine ratio at inclusion was correlated with significantly shorter OS and PFS, whereas no other biomarkers were found to be significant. The IL-6/IL-1Ra ratio reflects the imbalance between pro- and anti-inflammatory status in the plasma of patients and may be associated with tumor inflammatory status and the therapeutic outcome. The present study highlights the identification of the IL-6/IL-1Ra ratio as a biomarker of poor prognosis in terms of response to chemotherapy in two independent clinical studies.

\section{Introduction}

Lung cancer is the most frequently diagnosed cancer in China and the leading cause of cancer-related mortality (1). Non-small cell lung cancer (NSCLC) is the most common type of lung cancer. Despite advances in detection and treatment modalities, NSCLC is often diagnosed at an advanced stage and patients with stage IV disease have a poor prognosis (2). Platinum-based combination chemotherapy was the main therapeutic option for patients with metastatic NSCLC without epidermal growth factor receptor gene (EGFR) mutations prior to the emergence of Immune Checkpoint Inhibitor (ICI) therapies; however, the response to platinum-based chemotherapy may vary among patients with similar clinical characteristics, and resistance to chemotherapy is associated with a poor prognosis. NSCLC patients have a short progression-free survival (PFS) of only 3-5 months and a median overall survival (OS) of 7-12 months $(2,3)$.

The development of ICI-based therapies, alone or in combination with chemotherapy, appears to be promising for the treatment of late-stage cancer at diagnosis (4). These immunotherapeutic treatments are based on the immune status of the tumor (or the tumor microenvironment), as they can target programmed death-ligand 1 (PD-L1) expression on tumor cells (5), or induce the activation of effector immune cells, such as cytotoxic T cells (6). Retrospectively, numerous 
studies demonstrated that the immune status of the patient is crucial, as it may affect treatment outcome $(7,8)$. In addition, recent studies demonstrated that chemotherapy clearly affects immune effectors, such as PD-L1 expression and/or tumor-infiltrating lymphocyte (TIL) infiltration $(9,10)$.

One possible hypothesis explaining this association is the release of tumor-associated antigen and/or neo-antigen specific to the tumor, induced by the cytotoxicity of chemotherapies, and the priming of a specific immune response targeted against the tumor (11). These findings provide a rationale for the association of these multiple therapeutic options.

For both chemotherapy- and immune-based therapeutic strategies, there is a critical need for the identification of prognostic and predictive biomarkers to guide the selection of the therapeutic options for patients with metastatic NSCLC.

The tumor-based staging system and the Glasgow Prognostic Score assessed on systemic inflammatory response are commonly used to provide information on the best treatment options for individual patients with advanced NSCLC $(12,13)$; however, these systems are of limited predictive value in cases with metastatic (stage IV) NSCLC. New models have emerged for predicting the clinical outcome of chemotherapy-treated patients. In addition, with the introduction of ICI for cancer treatment, numerous immunophenotyping methods were developed to assess the tumor immune profile (14). These methods, mostly targeting in situ markers, are limited by the accessibility of the tumor site and the evolving immunophenotypic profile of the tumor over time and in response to chemotherapy- and/or immune-based therapies (15).

Circulating cytokines and chemokines are valuable biomarkers. A growing volume of evidence demonstrates that they play key roles in tumor progression, particularly cancer invasion and metastasis. Depending on these pro- or anti-inflammatory properties, cytokine levels in the blood are associated with tumor outcome (16), as is the case for interleukin (IL)-6, which appears to play a key role in the control of inflammation, irrespective of tumor type (17). Circulating cytokines are easily accessible for quantification and are more reflective of the heterogeneity of metastatic lung cancer compared with tissue biopsy; they provide useful information on the immuno-biological characteristics of the tumor and the systemic host immune response.

In the present study, Luminex bead-based multiplex assays (Bio-Rad Laboratories, Inc., Hercules, CA, USA) were used to measure the levels of 22 cytokines in the plasma of patients with metastatic NSCLC receiving platinum-based chemotherapy in a Chinese cohort from the Fudan University Shanghai Cancer Center (FUSCC) study. In this cohort, the impact of innate immune parameters (natural killer lymphocyte phenotype) on patient survival was previously analyzed (18). The objective of the present study was to evaluate various plasma cytokine levels, T-helper (Th)1/Th2 cytokine ratios, pro-/anti-inflammatory factor ratios and other patient characteristics as prognostic factors with a significant impact on OS and PFS. The identified parameters were also analyzed in patients from another clinical trial in Europe.

\section{Materials and methods}

Study design and patients. A cohort of Chinese patients enrolled in Fudan University (Shanghai, China), was analyzed to assess the plasma level of 22 cytokines. These cytokines were measured, in addition to the combined Th1/Th2 and pro-/anti-inflammatory cytokine ratios, in order to define a prognostic immune signature of chemotherapeutic treatment outcome in this Chinese patient cohort.

In the second part of the analysis, these prognostic immune signatures were analyzed in a cohort of European patients from another independent clinical trial.

The TG4010 study is registered with ClinicalTrials.gov no. NCT01383148, and the Fudan non-interventional study is registered as no. 101192-9 in the Fudan University Shanghai Cancer Center Ethical committee register.

\section{Study population}

Development cohort. A prospective monocentric observational study of 70 patients was conducted at the Department of Medical Oncology of FUSCC (Shanghai, China). Eligible patients were at least 18 years of age, with histologically or cytologically confirmed metastatic or recurrent metastatic NSCLC. The patients also had measurable disease according to the Response Evaluation Criteria in Solid Tumors version 1.0. Patients were considered ineligible if they had received previous systemic anticancer therapy $<1$ year prior to screening, or had severe drug allergies, active infection (fever at recruitment was a criterion for exclusion), other serious diseases or conditions, or inability to provide consent. Clinical data, including demographics (age and sex), clinicopathological characteristics [smoking status, Eastern Cooperative Oncology Group (ECOG) performance status, histological type, metastatic site and EGFR mutation status], chemotherapy treatments and laboratory parameters were collected prospectively (18). Patient recruitment lasted for 7 months and the patients were followed for OS and PFS during at least 2 years, with a median follow-up time of 28 months. The percentage of censoring was $50 \%$ for OS and $24.2 \%$ for PFS, as the dates of several patient deaths were unknown. EGFR mutation was defined as deletions on exon 19 or L858R mutations on exon 21. Other types of mutations or deletions were classified as wild-type for this analysis. The study protocol was approved by the Ethics Committee of FUSCC and written informed consent was obtained from all the participants.

Validation cohort. A retrospective study on the results of TG4010 clinical trial was conducted. The description of the clinical trial and the inclusion criteria were previously described (19). Validation was based on participants enrolled in the control arm of the TG4010 clinical trial, not treated with the cancer vaccine. These patients were followed-up for PFS and OS, with a median follow-up time of 37 months: The percentage of censoring was $12 \%$ for OS and none for PFS. In the validation cohort, attention was focused on the fact that the included patients did not have any infection-related pathologies at inclusion and fever was an exclusion criterion. Of note, 2 bronchitis, 1 laryngitis and 1 hepatitis B virus antibody-positive patients were reported.

Chemotherapy regimen and evaluation of response and follow-up. Eligible patients in the Chinese cohort received platinum-based first-line chemotherapy with cisplatin, 
carboplatin or lobaplatin, plus one of the following regimens: Taxane including docetaxel, paclitaxel or nab-paclitaxel, gemicitabine, pemetrexed and S-1 (oral fluoropyrimidine derivatives). The chemotherapy regimen was selected at the clinician's discretion based on the general condition of each individual patient. For the validation cohort, the patients received a similar platinum-based regimen including cisplatin and gemcitabine. For both studies, the regimen schedules were identical, comprising chemotherapy every 3 weeks, up to a total of 6 cycles or the appearance of progressive disease. The baseline clinical data were recorded at enrollment. OS was defined as the time from the initiation of therapy up to the date of the last follow-up or the date of death from any cause. Cases of survival with unknown status (patients without follow-up) or unknown date of death, the patients were censored (the cut-off date was set) at the date of last recorded contact. PFS was defined as the time from inclusion to disease progression or death. If the patient had progressed at the date of analysis, the progression date was defined as the date of last evaluable radiological assessment. For cases in which the tumor had not progressed or the patients were lost to follow-up, the patients were censored at the last evaluable radiological assessment.

Cytokine assays. Plasma was collected, aliquoted and frozen at $-70^{\circ} \mathrm{C}$ immediately after centrifugation and analyzed within 1 year. Cytokines were analyzed using Luminex $\mathrm{xMap}^{\mathrm{TM}}$ technology with Bio-Rad Bio-Plex 200 apparatus (Bio-Rad Laboratories, Inc.). The following cytokines, chemokines and growth factors were measured in the cohort samples: IL-1 $\beta$, IL-1Ra, IL-2, IL-4, IL-5, IL-6, IL-7, IL-8, IL-9, IL-10, IL-12 (p70), IL-15, IL-17, eotaxin, granulocyte colony-stimulating factor, granulocyte-macrophage colony-stimulating factor, interferon- $\gamma$, monocyte chemoattractant protein (MCP)-1, macrophage inflammatory protein (MIP)-1 $\alpha$, MIP-1 $\beta$, tumor necrosis factor (TNF)- $\alpha$ and vascular endothelial growth factor.

In addition, to assess more accurately the immune status related to the pathology, 27 cytokine ratios were calculated, as they are considered to reflect the balance between Th1 and Th2 cell types, as well as the pro- and anti-inflammatory status.

Markers of systemic inflammation. Absolute neutrophil count, absolute lymphocyte count, absolute platelet count and C-reactive protein (CRP) data obtained from medical records were registered from the date of the first chemotherapy treatment. The neutrophil/lymphocyte ratio, platelet/lymphocyte ratio (PLR) and CRP were used as indices of systemic inflammation.

Statistical analysis. Statistical analysis was performed with the non-parametric approach using Mann-Whitney and Fisher's exact tests for quantitative and qualitative variables, respectively.

A logistic model and the associated receiver operating characteristics (ROC) curve with 1-year survival as response was constructed for each cytokine or ratio to determine an optimal cut-off value. The point of ROC curve maximizing the Youden Index (sum of sensitivity and specificity) was determined as the optimal cut-off value (20). Candidate points were within the 1st and 3rd quartiles to provide balanced subgroups. This optimal cut-off value was used to dichotomize values into lowand high-level subgroups, and the same cut-off was used to define biomarker subgroups in the TG4010 validation cohort.

Survival curves for OS and PFS were generated using the Kaplan-Meier method, and log-rank tests were used for subgroup comparisons (low vs. high). To test the robustness of significant biomarkers, resampling with replacement was performed (1,000 iterations) and the log-rank test was applied for each sample: Significant biomarkers in $>50 \%$ of iterations were considered as robust. Significant robust biomarkers with optimal cut-off for OS and PFS were then tested in the TG4010 validation cohort. If relevant, hazard ratio (HR) was also estimated based on a Cox regression model including subgroup for each cytokine as covariate, and the median of PFS and OS with associated $95 \%$ confidence intervals (95\% CIs) were calculated.

Additionally, correlations between inflammatory markers and cytokines and ratios as continuous parameters were analyzed by calculation of the Spearman's rho.

Data handling and analysis were performed with SAS software, version 9.4 (SAS Institute, Inc., Cary, NC, USA). A P-value $<0.05$ was considered to indicate statistically significant differences.

\section{Results}

Characteristics of the Caucasian patient clinical trial compared with the Chinese FUSCC cohort. In order to validate the cytokine biomarkers analyzed in the FUSCC cohort, retrospective data from another independent study were used. Patients with similar clinical stages of NSCLC from a clinical trial performed in Europe (TG4010 trial) treated with a vaccinia virus expressing MUC-1 and IL-2 in combination with the SOC chemotherapeutic regimen, were investigated (19). Only the control arm receiving chemotherapy was used to analyze the relevance of the biomarkers identified in the Fudan study.

The two cohorts enrolled 138 patients with metastatic NSCLC, without radiotherapy, or at least 1 year after the last adjuvant treatment. The demographic and clinical characteristics of the patients are summarized in Table I. Considering the different ethnic populations, significant differences were identified between the FUSCC and the TG4010 cohorts in terms of sex, smoking history, ECOG score, CRP level and PLR at baseline, suggesting that patients from the TG4010 cohort were at higher risk. This was confirmed by a significantly lower PFS (the median PFS was 5.5 vs. 7.4 months, respectively; $\mathrm{P}<0.001$ ) and lower OS (the median OS was 10.8 vs. 19.4 months, respectively; $\mathrm{P}=0.002$ ) in the TG4010 cohort. In the FUSCC study, the median survival time was higher compared with previous reports, which may be explained by the smaller proportion of smokers (45.7 vs. $82.4 \%$, respectively) and lower CRP at baseline.

Of note, the difference in terms of tobacco use may be explained by the fact that, in the Chinese cohort, smoker status was defined at the time of inclusion only, and previous exposure was not taken into consideration, which was not the case in the TG4010 cohort. In addition, the effect of environmental pollution as the etiology of NSCLC in non-smoking Chinese patients was not excluded to explain this difference in proportions. 
Table I. Patient characteristics of metastatic non-small cell lung cancer cohorts.

\begin{tabular}{|c|c|c|c|c|}
\hline Demographic characteristics & FUSCC study $\mathrm{N}=70$ & TG4010 study $\mathrm{N}=68$ & Overall $\mathrm{N}=138$ & P-value \\
\hline Age & & & & 0.156 \\
\hline Mean (SD) & $57.0(9.33)$ & $60.2(8.08)$ & $58.6(8.85)$ & \\
\hline Median & 58.5 & 59 & 59 & \\
\hline Min-Max & $28.0-72.0$ & $37.0-78.0$ & $28.0-78.0$ & \\
\hline Q1-Q3 & $50.0-64.0$ & $55.0-67.0$ & $53.0-65.0$ & \\
\hline Sex & & & & 0.03 \\
\hline Male & $41(58.6 \%)$ & $52(76.5 \%)$ & $93(67.4 \%)$ & \\
\hline Female & $29(41.4 \%)$ & $16(23.5 \%)$ & $45(32.6 \%)$ & \\
\hline Smoking history & & & & $<0.001$ \\
\hline No & $32(45.7 \%)$ & $12(17.6 \%)$ & $44(31.9 \%)$ & \\
\hline Yes & $38(54.3 \%)$ & $56(82.4 \%)$ & $94(68.1 \%)$ & \\
\hline \multicolumn{5}{|l|}{ Disease characteristics } \\
\hline Histology & & & & 0.627 \\
\hline Missing & 3 & 7 & 10 & \\
\hline Adenocarcinoma & $58(86.6 \%)$ & $50(82.0 \%)$ & $108(84.4 \%)$ & \\
\hline Squamous cell carcinoma & $9(13.4 \%)$ & $11(18.0 \%)$ & $20(15.6 \%)$ & \\
\hline ECOG score & & & & 0.005 \\
\hline Missing & 1 & 2 & 3 & \\
\hline 0 & $13(18.8 \%)$ & $22(32.4 \%)$ & $35(25.5 \%)$ & \\
\hline 1 & $49(71.0 \%)$ & $46(67.6 \%)$ & $95(69.3 \%)$ & \\
\hline 2 & $7(10.1 \%)$ & $0(0.0 \%)$ & $7(5.1 \%)$ & \\
\hline \multicolumn{5}{|l|}{$E G F R$} \\
\hline Missing & 12 & & & \\
\hline Wild-type & $21(36.2 \%)$ & Not collected & & \\
\hline Mutation & $37(63.8 \%)$ & Not collected & & \\
\hline CRP level (mg/l) & & & & $<0.001$ \\
\hline Missing & 3 & 5 & 8 & \\
\hline$\leq 10$ & $45(67.2 \%)$ & $23(36.5 \%)$ & $68(52.3 \%)$ & \\
\hline$>10$ & $22(32.8 \%)$ & $40(63.5 \%)$ & $62(47.7 \%)$ & \\
\hline Platelets/lymphocyte ratio & & & & 0.003 \\
\hline Missing & 0 & 1 & 1 & \\
\hline$\leq 160$ & $37(52.9 \%)$ & $18(26.9 \%)$ & $55(40.1 \%)$ & \\
\hline$>160$ & $33(47.1 \%)$ & $49(73.1 \%)$ & $82(59.9 \%)$ & \\
\hline Neutrophil/lymphocyte ratio & & & & 0.111 \\
\hline Missing & 0 & 1 & 1 & \\
\hline$\leq 3$ & $31(44.3 \%)$ & $20(29.9 \%)$ & $51(37.2 \%)$ & \\
\hline$>3$ & $39(55.7 \%)$ & $47(70.1 \%)$ & $86(62.8 \%)$ & \\
\hline \multicolumn{5}{|l|}{ Survival data } \\
\hline Median follow-up (months) [95\% CI] & $27.6[27.2 ; 28.2]$ & $36.8[31.9 ; 40.9]$ & $29.1[27.6 ; 30.3]$ & $<0.001$ \\
\hline \multicolumn{5}{|l|}{ Overall survival (OS) } \\
\hline Number of events ( $\%$ censoring) & $35(50 \%)$ & $60(12 \%)$ & $95(31 \%)$ & \\
\hline Median OS (months) $[95 \% \mathrm{CI}]$ & $19.4[13.2]$ & $10.8[8.3 ; 12.5]$ & $12.7[11.3 ; 16.3]$ & 0.0015 \\
\hline \multicolumn{5}{|l|}{ Progression free survival } \\
\hline Number of events ( $\%$ censoring) & $53(24.2 \%)$ & $68(0 \%)$ & $121(12.3 \%)$ & \\
\hline Median PFS (months) [95\% CI] & $7.4[4.6 ; 10.6]$ & $5.5[4.3 ; 6.2]$ & $5.8[4.7 ; 6.5]$ & $<0.001$ \\
\hline
\end{tabular}

Continuous variables were compared with a Wilcoxon-Mann-Whitney test, categorical variables with a Fisher's exact test and survival variables with a log-rank test. Bold values highlight statistically significant data with $\mathrm{P}<0.05$. CI, confidence interval; N, number of patients; PFS, progression-free survival; $\mathrm{SD}$, standard deviation; OS, overall survival; EGFR, epidermal growth factor receptor; CRP, C-reactive protein; ECOG, Eastern Cooperative Oncology Group; FUSCC, Fudan University Shanghai Cancer Center. 
A

Chinese cohort - OS

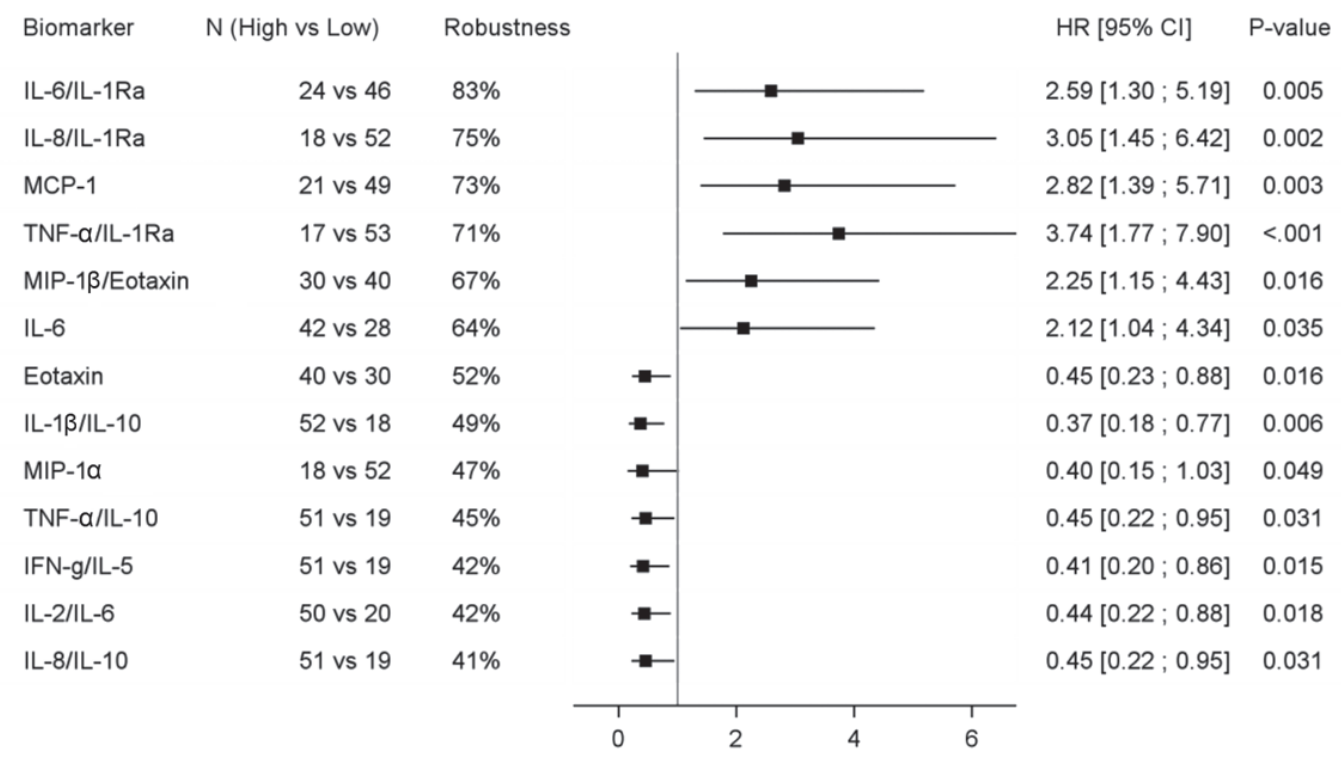

B

Biomarker
IL-6/IL-1Ra
IL-8/IL-1Ra
MCP-1
TNF- $\alpha / / L-1 R a$
MIP-1 $\beta / E$ Etaxin
Eotaxin

Chinese cohort - PFS

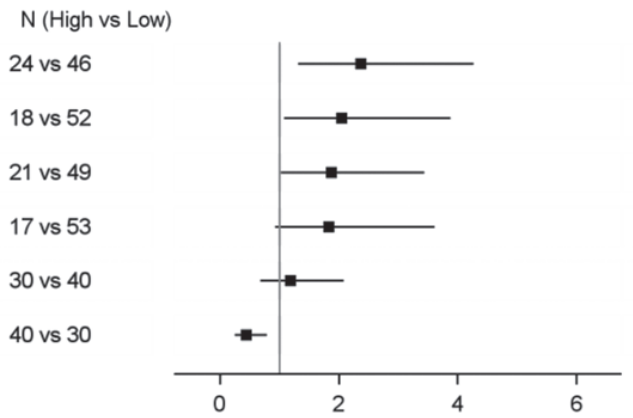

$\begin{array}{cc}\text { HR }[95 \% \mathrm{Cl}] & \text { P-value } \\ 2.37[1.32 ; 4.27] & 0.003 \\ 2.04[1.08 ; 3.88] & 0.025 \\ 1.88[1.02 ; 3.44] & 0.038 \\ 1.83[0.93 ; 3.61] & 0.075 \\ 1.19[0.68 ; 2.08] & 0.549 \\ 0.44[0.25 ; 0.79] & 0.005\end{array}$

Figure 1. Forest plot presenting log-rank tests and HR of cytokines or ratios for OS and PFS in the FUSCC study. (A) A total of 13 biomarkers were identified as significant in chemotherapeutic response with OS. (B) A total of 6 of 13 were found to be robust using the resampling approach, whereas only 4 were still found to be significantly associated with PFS. FUSCC, Fudan University Shanghai Cancer Center; OS, overall survival; PFS, progression-free survival; N, number of patients; HR, hazard ratio; CI, confidence interval; MCP, monocyte chemoattractant protein; TNF, tumor necrosis factor; MIP, macrophage inflammatory protein; IL, interleukin.

Cytokine profile analysis in the FUSCC cohort. A total of 13 cytokines or ratios were found to be significantly associated with OS in the FUSCC study using Kaplan-Meier curves and log-rank tests. Among these 13 biomarkers (Fig. 1A), only 6 were found to be sufficiently robust using the resampling approach. Patients with high values of IL-6/IL-1Ra $(\mathrm{HR}=2.59$, $\mathrm{P}=0.005)$, IL-8/IL-1Ra $(\mathrm{HR}=3.05, \mathrm{P}=0.002)$, MCP-1 $(\mathrm{HR}=2.82, \mathrm{P}=0.003), \mathrm{TNF}-\alpha / \mathrm{IL}-1 \mathrm{Ra}(\mathrm{HR}=3.74, \mathrm{P}<0.001)$ and MIP-1 $\beta /$ eotaxin $(\mathrm{HR}=2.01, \mathrm{P}=0.038)$ had a significantly shorter $\mathrm{OS}$, whereas patients with high levels of eotaxin $(\mathrm{HR}=0.45$, $\mathrm{P}=0.016$ ) were found to have a significantly longer OS.

These significant robust biomarkers were evaluated for the PFS analysis and it was observed that only IL-6/IL-1Ra $(\mathrm{HR}=2.37$, $\mathrm{P}=0.003), \mathrm{IL}-8 / \mathrm{IL}-1 \mathrm{Ra}$ (HR=2.04, $\mathrm{P}=0.025), \mathrm{MCP}-1 \quad(\mathrm{HR}=1.88$, $\mathrm{P}=0.038)$ and eotaxin $(\mathrm{HR}=0.44, \mathrm{P}=0.005)$ were significantly associated (Fig. 1B) with PFS, as was observed with OS.

Validation of biomarker relevance using the TG4010 cohort. Using the defined optimal cut-off obtained in the
Chinese cohort, robust biomarkers were analyzed for the TG4010 cohort. Only three biomarkers had an acceptable subgroup sample size for comparisons using these cut-offs, and only IL-6/IL-1Ra was also found to be significant in the validation cohort. Patients in the TG4010 cohort with increased IL-6/IL-1Ra ratio at inclusion were also found to have a significantly lower $\mathrm{OS}(\mathrm{HR}=2.29, \mathrm{P}=0.006)$ and $\mathrm{PFS}$ (HR=2.42, $\mathrm{P}=0.004$ ) (Table II and Fig. 2).

As regards the three biomarkers not used for validation due to the insufficient sample size (MCP-1, eotaxin and TNF- $\alpha / \mathrm{IL}-1 \mathrm{Ra}$ ), a new Youden optimal cut-off was defined to investigate the association with OS and PFS in the TG4010 cohort; however, even with a new optimal cut-off, the subgroups were not found to be associated with either OS or PFS (data not shown).

Analysis of IL-6 and IL1-Ra cytokine levels independently in the two clinical cohorts. IL-6 and IL1-Ra levels were analyzed independently in the two cohorts. Only a high IL-6/IL1-Ra level was found to be significantly associated 
Table II. Statistical analysis of the robust cytokines level impact on OS and PFS in the TG4010 cohort.

\begin{tabular}{|c|c|c|c|c|c|}
\hline \multirow[b]{2}{*}{ Biomarker } & \multirow[b]{2}{*}{ N (High vs. Low) } & \multicolumn{2}{|c|}{ OS } & \multicolumn{2}{|c|}{ PFS } \\
\hline & & P-value & $\mathrm{HR}[95 \% \mathrm{CI}]$ & P-value & $\mathrm{HR}[95 \% \mathrm{CI}]$ \\
\hline IL-6/IL-1Ra & 14 vs. 54 & 0.006 & $2.29[1.25 ; 4.19]$ & 0.004 & $2.42[1.30 ; 4.48]$ \\
\hline IL-8/IL-1Ra & 40 vs. 28 & 0.335 & $1.29[0.77 ; 2.17]$ & 0.419 & $1.22[0.75 ; 2.00]$ \\
\hline MCP-1 & 64 vs. 4 & Not done & & Not done & \\
\hline TNF- $\alpha /$ IL-1Ra & 0 vs. 68 & Not done & & Not done & \\
\hline MIP-1 $\beta /$ Eotaxin & 38 vs. 30 & 0.217 & $1.38[0.83 ; 2.30]$ & 0.137 & $1.44[0.88 ; 2.35]$ \\
\hline Eotaxin & 3 vs. 65 & Not done & & Not done & \\
\hline
\end{tabular}

P-values were from log-rank test and HR from Cox model including low and high level subgroups of cytokines as covariate. Bold values highlight statistically significant data with $\mathrm{P}<0.05$. CI, confidence interval; N, number of patients; PFS, progression-free survival; HR, hazard ratio; OS, overall survival; MCP, monocyte chemoattractant protein; TNF, tumor necrosis factor; MIP, macrophage inflammatory protein; IL, interleukin.
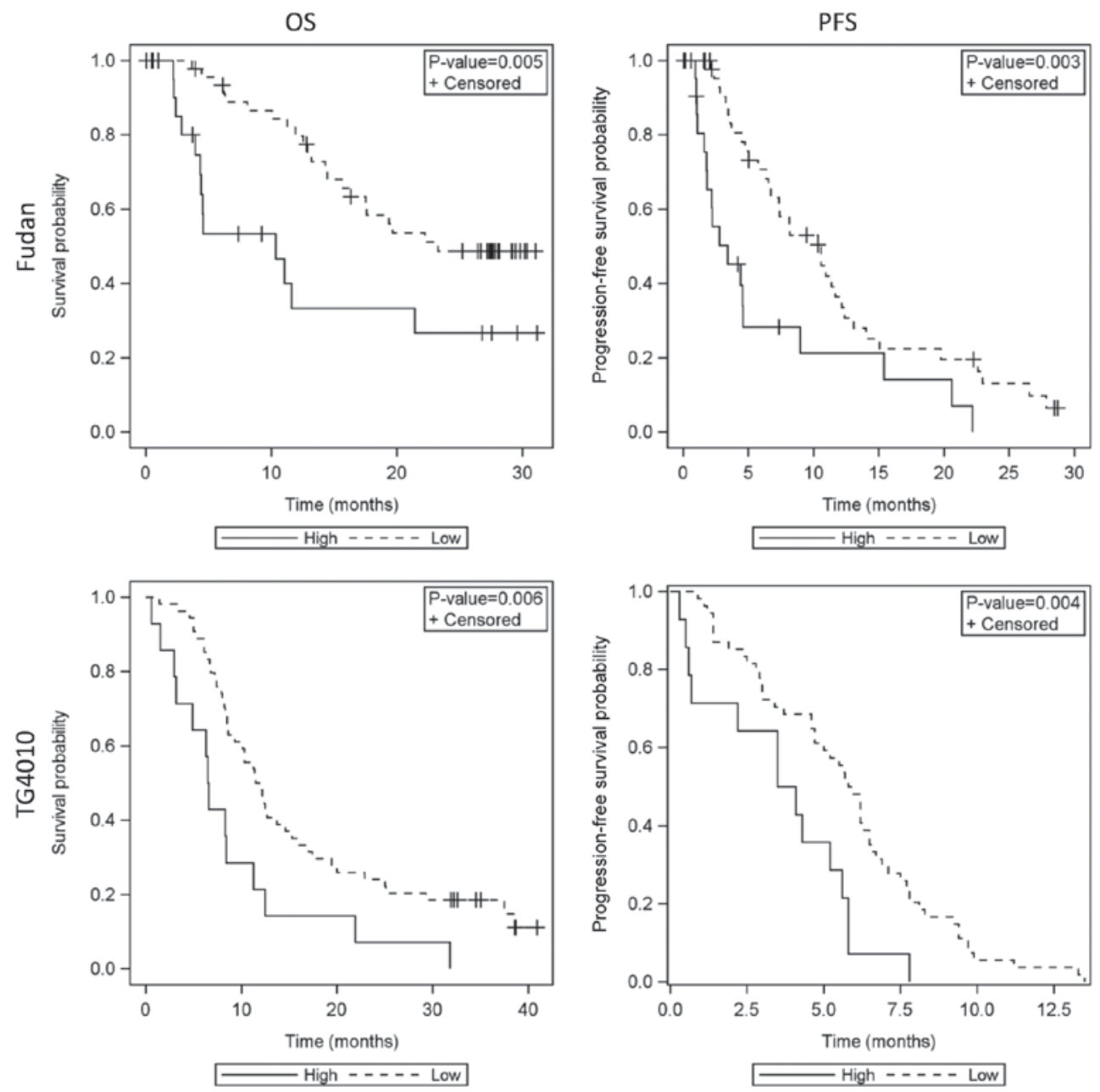

Figure 2. Kaplan-Meier curves and log-rank tests for the prognostic IL-6/IL-1Ra biomarker in terms of OS and PFS for the FUSCC and TG4010 cohorts. FUSCC, Fudan University Shanghai Cancer Center; OS, overall survival; PFS, progression-free survival; Il, interleukin.

with an unfavorable prognosis, with shorter OS and PFS in both studies (Table III). In the validation cohort (TG4010), a high IL-6 level was found to be significantly associated with shorter OS $(\mathrm{P}<0.001)$, but the association between IL- 6 level and PFS was not statistically significant $(\mathrm{P}=0.051)$. IL-6, as a marker of the inflammation status, was prognostic of weak response to chemotherapy in that study, in which patients had more advanced disease.

This was not the case in the Chinese cohort, in which the association of high IL-6 level with OS and PFS was not 
Table III. Statistical analysis of IL-6 and IL-1ra levels versus OS and PFS.

\begin{tabular}{|c|c|c|c|c|c|}
\hline \multirow[b]{2}{*}{ Biomarker } & \multirow[b]{2}{*}{ N (High vs. Low) } & \multicolumn{2}{|c|}{ OS } & \multicolumn{2}{|c|}{ PFS } \\
\hline & & P-value & $\mathrm{HR}[95 \% \mathrm{CI}]$ & P-value & $\mathrm{HR}[95 \% \mathrm{CI}]$ \\
\hline \multicolumn{6}{|c|}{ A, Chinese cohort } \\
\hline IL-6 & 43 vs. 27 & 0.067 & $1.92[0.942 ; 3.934]$ & 0.569 & $1.18[0.671 ; 2.064]$ \\
\hline IL-1Ra & 37 vs. 33 & 0.526 & $0.81[0.42 ; 1.57]$ & 0.2654 & $0.73[0.42 ; 1.27]$ \\
\hline IL-6/IL-1Ra & 24 vs. 46 & 0.005 & $2.59[1.30 ; 5.19]$ & 0.003 & $2.37[1.32 ; 4.27]$ \\
\hline
\end{tabular}

B, TG4010 cohort

\begin{tabular}{llllll}
\hline IL-6 & 25 vs. 43 & 0.051 & $1.68[0.99 ; 2.84]$ & $<.001$ & $2.51[1.45 ; 4.34]$ \\
IL-1Ra & 38 vs. 30 & 0.544 & $1.17[0.70 ; 1.96]$ & 0.337 & $1.27[0.77 ; 2.07]$ \\
IL-6/IL-1Ra & 14 vs. 54 & $\mathbf{0 . 0 0 6}$ & $2.29[1.25 ; 4.19]$ & $\mathbf{0 . 0 0 4}$ & $2.42[1.30 ; 4.48]$ \\
\hline
\end{tabular}

P-values were from log-rank test and HR from Cox model including low and high-level subgroups of cytokines as covariate. Bold values highlight statistically significant data with $\mathrm{P}<0.05$. CI, confidence interval; $\mathrm{N}$, number of patients; PFS, progression-free survival; HR, hazard ratio; OS, overall survival; IL, interleukin.

Table IV. Association of IL-6, IL-1Ra and IL-6/IL-1Ra with clinical characteristics.

\begin{tabular}{|c|c|c|c|c|c|c|}
\hline \multirow[b]{2}{*}{ Variable } & \multicolumn{3}{|c|}{ FUSCC study $(\mathrm{N}=70)$} & \multicolumn{3}{|c|}{ TG4010 study $(\mathrm{N}=68)$} \\
\hline & IL-6/IL-1Ra & IL-6 & IL-1Ra & IL-6/IL-1Ra & IL-6 & IL-1Ra \\
\hline Age $(\leq 60$ vs. $>60)$ & 0.096 & 0.278 & 0.097 & 0.095 & 0.002 & 0.015 \\
\hline Sex (M vs. F) & 0.017 & 0.893 & 0.096 & 0.23 & 0.069 & 0.159 \\
\hline Smoking status ( $\mathrm{Y}$ vs. N) & 0.013 & 0.92 & 0.137 & 0.0371 & 0.249 & 0.278 \\
\hline $\operatorname{CRP}(\leq 10$ vs. $>10)$ & 0.001 & $<0.001$ & 0.052 & 0.002 & $<0.001$ & 0.062 \\
\hline $\operatorname{ECOG}(0,1$ and $2 / 3)$ & 0.092 & 0.17 & 0.523 & 0.199 & 0.297 & 0.829 \\
\hline
\end{tabular}

Comparisons done with non-parametric Mann-Whitney test (Kruskal-Wallis test for ECOG) and biomarkers were treated as continuous variables. Bold values highlight statistically significant data with $\mathrm{P}<0.05$. N, number of patients; M, Male; F, Female; Y, Yes; N, No; CRP, C-reactive protein; ECOG, Eastern Cooperative Oncology Group; IL, interleukin; FUSCC, Fudan University Shanghai Cancer Center.

significant $(\mathrm{P}=0.067$ and $\mathrm{P}=0.569$ respectively). IL1-Ra was not found to be significantly associated with either OS or PFS in neither of the two cohort.

Association with inflammation and other clinical parameters. The association between the IL-6/IL-1Ra ratio (as a continuous variable) and independent cytokine levels with certain clinical parameters known to be associated with inflammatory status, such as CRP, smoking and ECOG performance status, was then analyzed. In the Chinese cohort, male smokers with an elevated CRP level ( $>10 \mathrm{mg} / \mathrm{l})$ were found to have a higher IL-6/IL-1Ra ratio. In the TG4010 cohort, the same result was observed for smokers with an elevated CRP level (Table IV). The Spearman's rho between IL-6/IL-1Ra ratio and CRP at baseline was significantly different from 0 , with a correlation coefficient of 0.43 and 0.44 in the Chinese and TG4010 cohorts, respectively (data not shown).
For IL-6 alone, significantly higher values were found in the subgroup of patients with elevated CRP level in both cohorts. Moreover, in the TG4010 cohort, patients aged $>60$ years were found to have both higher IL-6 and IL-1Ra levels.

\section{Discussion}

In the present study, the circulating cytokine levels and their ratios were investigated in patients with metastatic NSCLC as prognostic indicators for OS and PFS. Among 22 individual cytokines and 27 cytokine ratios, only the IL-6/IL-1Ra ratio, a pro-/anti-inflammatory ratio, was found to be a predictor for survival outcome in both the Chinese cohort and the validation Caucasian cohort. A high value of the IL-6/IL-1Ra ratio was associated with shorter survival, indicating that, despite the two cohorts differing in several parameters, such as ethnicity, disease stage, ECOG performance status and smoking history, 
this biomarker remained prognostic of poor PFS and OS. It would be of great clinical interest to study this biomarker in other cohorts of NSCLC patients under chemotherapy, or with other types of cancer.

IL-6, a pleiotropic pro-inflammatory Th2 cytokine, has been demonstrated by several studies to participate in tumor cell proliferation, apoptosis and angiogenesis $(21,22)$. It was also reported that high IL-6 expression in patient plasma was associated with shorter OS in lung (23) and colorectal cancer (24). In addition, IL-6 alone was found to be significantly associated with PFS in the TG4010 study. In the FUSCC cohort, the fact that IL- 6 was not correlated with shorter PFS may be explained by a better clinical status of the patients in that study, with an expected less prominent inflammatory tumor status. By contrast, IL-1Ra, an anti-inflammatory cytokine, has been shown to decrease tumor growth, angiogenesis and metastasis in murine xenograft models (25). In the present study, the levels of either cytokine (IL-6 or IL-1Ra) considered independently were not found to be significantly associated with the clinical outcome of NSCLC in neither of the two cohorts, whereas the IL-6/IL-1Ra ratio was identified as an independent prognostic biomarker for PFS and OS in NSCLC.

The ratio of pro- and anti-inflammatory cytokines is a marker of an imbalance in the regulation of the inflammation that occurs during oncogenesis. Inflammation is considered as a condition that favors cancer development, and also as a condition that arises during oncogenic changes in cancer cells (26). The molecular mechanisms underlying the inflammatory process and cancer development are poorly understood. Emerging data highlight the important pleiotropic roles of intratumoral cytokines in cancer development (27).

In the present study, we investigated the correlation of this biomarker with other common clinical parameters and inflammation. As regards PFS and OS, the IL-6/IL-1Ra ratio was associated with the CRP level, but not with smoking or ECOG status. This correlation of the IL-6/IL-1Ra ratio with tumor inflammation offers the possibility to access the tumor status using these easily accessible peripheral cytokine levels as diagnostic biomarkers more conveniently, as compared with intratumoral cytokine evaluation.

Blood cytokines are particularly adapted for tracking and monitoring treatment response and disease progression. In addition, the inflammatory tumor status is important for therapeutic decision-making, for example when considering immunotherapeutic products. It should be of interest to analyze the correlation between this biomarker and the immunophenotype of the tumor, such as TIL infiltration and/or intratumoral IL expression by immunohistochemistry analysis.

The cytokine levels and ratios in NSCLC patients treated with the immunotherapeutic vaccine from the TG4010 clinical trial were also analyzed. This immunotherapeutic treatment is based on a viral vector expressing the MUC-1 tumor antigen, with the aim of activating the immune response against MUC-1-expressing tumors. Interestingly, in patients treated with TG4010 immunotherapy, the IL-6/IL-1Ra ratio was not found to be associated with a significant difference in either PFS or OS (data not shown). Considering that an immunotherapeutic vaccine should re-program the immune status and/or the response against tumor cells, the IL-6/IL-1Ra ratio is not associated with poor prognosis in this therapeutic context (19). This observation suggests that the IL-6/IL-1Ra ratio may be a valuable biomarker of the immune system status (within the tumor) in the context of chemotherapy to predict the efficacy of treatment or redirect the selection of therapeutic options.

In the future, the development of cytokine biomarkers may enable the introduction of an accurate correlative prognostic or predictive signature to determine the best therapeutic option for each patient.

\section{Acknowledgements}

The authors would like to sincerely thank Dr Jianhua Chang and Dr Xianghua Wu (Department of Medical Oncology, Fudan University, Shanghai Cancer Center, Shanghai, China) for contributing their clinical expertise in the follow-up of Fudan patients. The authors would also like to thank Dr Laurence Zitvogel, Dr Guido Kroemer (who both work at Gustave Roussy Institut, Villejuife, France), Dr Alexandra Gentry-Maharaj (MRC Clinical Trials Unit, University College of London, London, United Kingdom) and Dr Manish S. Patankar (University of Wisconsin, UW Hospital and Clinics, Madison, WI, USA) for their excellent advice. Finally, the authors would also like to thank Dr Caroline Schenkels (Transgene S.A., Illkirchn, France) for her help in the redaction of this paper.

\section{Funding}

This study was supported by Transgene S.A., France, and by a grant from the Shanghai Municipal Commission of Health and Family Planning (grant no. 201440423). It has also benefited from the expertise of the IMMUNOCAN network (project FP7-INCO-2011-7 funded by European Commission, project ID: 294929) for cytokine detection.

\section{Availability of data and materials}

The datasets used and/or analyzed during the current study are available from the corresponding author on reasonable request.

\section{Authors' contributions}

FL was involved in data collection, performed cytokine measurements and participated in analyzing and interpreting the results. BS performed statistical analyses, XP and RG helped in cytokine detection method development, JW and HY participated in patient recruitment and data collection, XM and RM participated in the Fudan trial design and scientific discussions, LB participated in data analysis and scientific discussions. FL, $\mathrm{BS}$ and LB were major contributors to writing this manuscript.

\section{Ethics approval and consent to participate}

The Fudan study was approved by the Ethics Committee of FUSCC, and the TG4010 study was approved by all national health authorities and Ethics Committees of the institutions in which the trial was conducted. Written informed consent was obtained from all the patients who participated in the Fudan and TG4010 studies. 


\section{Patient consent for publication}

Written informed consent was obtained from all the patients who participated in the Fudan and TG4010 studies. All data were anonymized to prevent patient identification.

\section{Competing interests}

FL, JW, HY and XM declare that they have no competing interests. BS, XP, RG, RM and LB were employees of Transgene at the time the study was conducted.

\section{References}

1. Chen W, Zheng R, Baade PD, Zhang S, Zeng H, Bray F, Jemal A, Yu XQ and He J: Cancer statistics in China, 2015. CA Cancer J Clin 66: 115-132, 2016.

2. Scagliotti G, Hanna N, Fossella F, Sugarman K, Blatter J, Peterson P, Simms L and Shepherd FA: The differential efficacy of pemetrexed according to NSCLC histology: A review of two phase III studies. Oncologist 14: 253-263, 2009.

3. Scagliotti GV, Parikh P, von Pawel J, Biesma B, Vansteenkiste J, Manegold C, Serwatowski P, Gatzemeier U, Digumarti R, Zukin M, et al: Phase III study comparing cisplatin plus gemcitabine with cisplatin plus pemetrexed in chemotherapy-naive patients with advanced-stage non-small-cell lung cancer. J Clin Oncol 26: 3543-3551, 2008.

4. Santabarbara G, Maione P, Rossi A, Palazzolo G and Gridelli C: Novel immunotherapy in the treatment of advanced non-small cell lung cancer. Expert Rev Clin Pharmacol 9: 1571-1581, 2016.

5. Daud AI, Wolchok JD, Robert C, Hwu WJ, Weber JS, Ribas A, Hodi FS, Joshua AM, Kefford R, Hersey P, et al: Programmed death-ligand 1 expression and response to the anti-programmed death 1 antibody pembrolizumab in melanoma. J Clin Oncol 34 4102-4109, 2016.

6. Bremnes RM, Busund LT, Kilvær TL, Andersen S, Richardsen E, Paulsen EE, Hald S, Khanehkenari MR, Cooper WA, Kao SC and Dønnem T: The role of tumor-infiltrating lymphocytes in development, progression, and prognosis of non-small cell lung cancer. J Thorac Oncol 11: 789-800, 2016.

7. Teng F, Meng X, Wang X, Yuan J, Liu S, Mu D, Zhu H, Kong L and Yu J: Expressions of CD8+TILs, PD-L1 and Foxp3+TILs in stage I NSCLC guiding adjuvant chemotherapy decisions. Oncotarget 7: 64318-64329, 2016.

8. McLemore LE, Janakiram M, Albanese J, Shapiro N, Lo Y, Zang $X$ and Fineberg S: An immunoscore using PD-L1, CD68, and Tumor-infiltrating Lymphocytes (TILs) to predict response to neoadjuvant chemotherapy in invasive breast cancer. App Immunohistochem Mol Morphol 26: 611-619, 2018.

9. Zhang P, Ma Y, Lv C, Huang M, Li M, Dong B, Liu X, An G, Zhang $\mathrm{W}$, Zhang $\mathrm{J}$, et al: Up-regulation of programmed cell death ligand 1 promotes resistance response in non-small cell lung cancer patients with neo-adjuvant chemotherapy. Cancer Sci 107: 1563-1571, 2016

10. Mesnage SJL, Auguste A, Genestie C, Dunant A, Pain E, Drusch F, Gouy S, Morice P, Bentivegna E, Lhomme C, et al: Neoadjuvant chemotherapy (NACT) increases immune infiltration and programmed death-ligand 1 (PD-L1) expression in epithelial ovarian cancer (EOC). Ann Oncol 28: 651-657, 2017.
11. Vacchelli E, Aranda F, Eggermont A, Galon J, Sautès-Fridman C, Cremer I, Zitvogel L, Kroemer G and Galluzzi L: Trial watch: Chemotherapy with immunogenic cell death inducers. Oncoimmunology 3: e27878, 2014.

12. Leung EY, Scott HR and McMillan DC: Clinical utility of the pretreatment glasgow prognostic score in patients with advanced inoperable non-small cell lung cancer. J Thorac Oncol 7: 655-662, 2012.

13. Proctor MJ, Morrison DS, Talwar D, Balmer SM, O'Reilly DS, Foulis AK, Horgan PG and McMillan DC: An inflammation-based prognostic score (mGPS) predicts cancer survival independent of tumour site: A glasgow inflammation outcome study. Br J Cancer 104: 726-734, 2011.

14. Kalra J and Baker J: Multiplex immunohistochemistry for mapping the tumor microenvironment. Methods Mol Biol 1554: 237-251, 2017.

15. Bindea G, Mlecnik B, Tosolini M, Kirilovsky A, Waldner M, Obenauf AC, Angell $\mathrm{H}$, Fredriksen $\mathrm{T}$, Lafontaine L, Berger A, et al: Spatiotemporal dynamics of intratumoral immune cells reveal the immune landscape in human cancer. Immunity 39: 782-795, 2013.

16. Lippitz BE: Cytokine patterns in patients with cancer: A systematic review. Lancet Oncol 14: e218-e228, 2013.

17. Lippitz BE and Harris RA: Cytokine patterns in cancer patients: A review of the correlation between interleukin 6 and prognosis. Oncoimmunology 5: e1093722, 2016.

18. Yu H, Liu F, Sansas B, Kang B, Preville X, Wu X, Chang J, Micol R, Wang J and Meng X: Typing of killer-cell immunoglobulin-like receptors and their cognate human leukocyte antigen class I ligands predicts survival of Chinese Han patients with metastatic non-small-cell lung cancer. Mol Clin Oncol 6: 279-285, 2017.

19. Quoix E, Ramlau R, Westeel V, Papai Z, Madroszyk A, Riviere A, Koralewski P, Breton JL, Stoelben E, Braun D, et al: Therapeutic vaccination with TG4010 and first-line chemotherapy in advanced non-small-cell lung cancer: A controlled phase 2B trial. Lancet Oncol 12: 1125-1133, 2011.

20. Fluss R, Faraggi D and Reiser B: Estimation of the Youden Index and its associated cutoff point. Biom J 47: 458-472, 2005.

21. Naugler WE and Karin M: The wolf in sheep's clothing: The role of interleukin-6 in immunity, inflammation and cancer. Trends Mol Med 14: 109-119, 2008.

22. Yu H, Lee H, Herrmann A, Buettner R and Jove R: Revisiting STAT3 signalling in cancer: New and unexpected biological functions. Nat Rev Cancer 14: 736-746, 2014.

23. Wang YS, Miao LY, Liu L, Cai HR, Ding JJ, Ren SX, Zhou CC and Schmid-Bindert G: Serum cytokine levels in patients with advanced non-small cell lung cancer: Correlation with clinical outcome of erlotinib treatment. Chin Med J (Engl) 126: 3931-3935, 2013.

24. Wang Z, Wu P, Wu D, Zhang Z, Hu G, ZhaoS, Lai Y and Huang J: Prognostic and clinicopathological significance of serum interleukin-6 expression in colorectal cancer: A systematic review and meta-analysis. Onco Targets Ther 8: 3793-3801, 2015.

25. Lewis AM, Varghese $\mathrm{S}, \mathrm{Xu} \mathrm{H}$ and Alexander HR: Interleukin-1 and cancer progression: The emerging role of interleukin-1 receptor antagonist as a novel therapeutic agent in cancer treatment. J Transl Med 4: 48, 2006.

26. Mantovani A, Allavena P, Sica A and Balkwill F: Cancer-related inflammation. Nature 454: 436-444, 2008.

27. Landskron G, De la Fuente M, Thuwajit P, Thuwajit $C$ and Hermoso MA: Chronic inflammation and cytokines in the tumor microenvironment. J Immunol Res 2014: 149185, 2014. 